\title{
Microwave ablation for the management of pulmonary inflammatory myofibroblastic tumor: a case report and literature review
}

\author{
Yizhong Zhang ${ }^{1}$, Guoping Zheng ${ }^{1}$, Xiaopeng Meng ${ }^{1}$, Yuanjing Li $^{1}$, Dan Shi ${ }^{2}$, Jinna Yu ${ }^{3}$ \\ ${ }^{1}$ Department of Cardiothoracic Surgery, Shaoxing Second Hospital, Shaoxing, China; ${ }^{2}$ Department of Pathology, Shaoxing Second Hospital, \\ Shaoxing, China; ${ }^{3}$ Department of Radiology, Shaoxing Second Hospital, Shaoxing, China \\ Correspondence to: Yizhong Zhang. Department of Cardiothoracic Surgery, Shaoxing Second Hospital, Shaoxing 312000 , China. \\ Email: sxeyxwkzyz@163.com.
}

\begin{abstract}
Inflammatory myofibroblastic tumor (IMT) is a rare mesenchymal tumor. Although IMT generally exhibits benign biological behavior, some IMT patients may develop local recurrence or even distant metastasis. Surgery is the most common therapeutic approach. However, additional treatment options are necessary for those who are unable to undergo surgical treatments. Microwave ablation (MWA) is a developing treatment option for unresectable lung cancer. Compared with radiofrequency ablation (RFA), MWA possesses many advantages including larger ablation zones, shorter heating times, and less susceptibility to heat sink. Herein, we reported the case of an 80-year-old male patient who presented with a mass in the right lower pulmonary lobe and right pleural effusion. The maximum diameter of the mass was $53.76 \mathrm{~mm}$, and the patient's main complaint was fatigue for 2 months. Blood test showed severe anemia (hemoglobin: $79 \mathrm{~g} / \mathrm{L}$ ) and hypoproteinemia (albumin: $27.7 \mathrm{~g} / \mathrm{L}$ ). After undergoing blood transfusion, supplementation of albumin, thoracical puncturing and piping, the patient's nutritional condition was improved (hemoglobin, $95 \mathrm{~g} / \mathrm{L}$; serum albumin, $29.9 \mathrm{~g} / \mathrm{L}$ ). The pathological diagnosis was pulmonary IMT by percutaneous lung biopsy. The patient was successfully treated with three rounds of percutaneous MWA and has no evidence of recurrence nearly 3 years later. This case has expanded the therapeutic options for elderly patients with pulmonary IMT. Percutaneous MWA monotherapy might serve as an emerging treatment strategy for medically inoperable patients.
\end{abstract}

Keywords: Microwave ablation (MWA); computed tomography-guided ablation (CT-guided ablation); inflammatory myofibroblastic tumor (IMT); lung tumor; case report

Submitted Aug 30, 2021. Accepted for publication Sep 22, 2021.

doi: $10.21037 /$ tcr-21-1885

View this article at: https://dx.doi.org/10.21037/tcr-21-1885

\section{Introduction}

Inflammatory myofibroblastic tumor (IMT) is a distinctive lesion composed of myofibroblastic and fibroblastic spindle cells accompanied by an inflammatory infiltration of plasma cells, lymphocytes, and eosinophils. IMT typically exhibits benign clinical behavior, although aggressive behavior and recurrences occur occasionally (1). IMT has been reported to occur in multiple anatomical locations, including the abdominopelvic region, lung, retroperitoneum, bone, somatic soft tissues, and central nervous system (2). It has thus far been referred to as an inflammatory pseudotumor, plasma cell granuloma, inflammatory myofibrohistiocytic proliferation, inflammatory fibrosarcoma, etc. (3). IMT is a rare mesenchymal tumor that mainly affects children and young adults. However, it is also seen among elderly men (4).

^ ORCID: 0000-0002-2893-6834. 

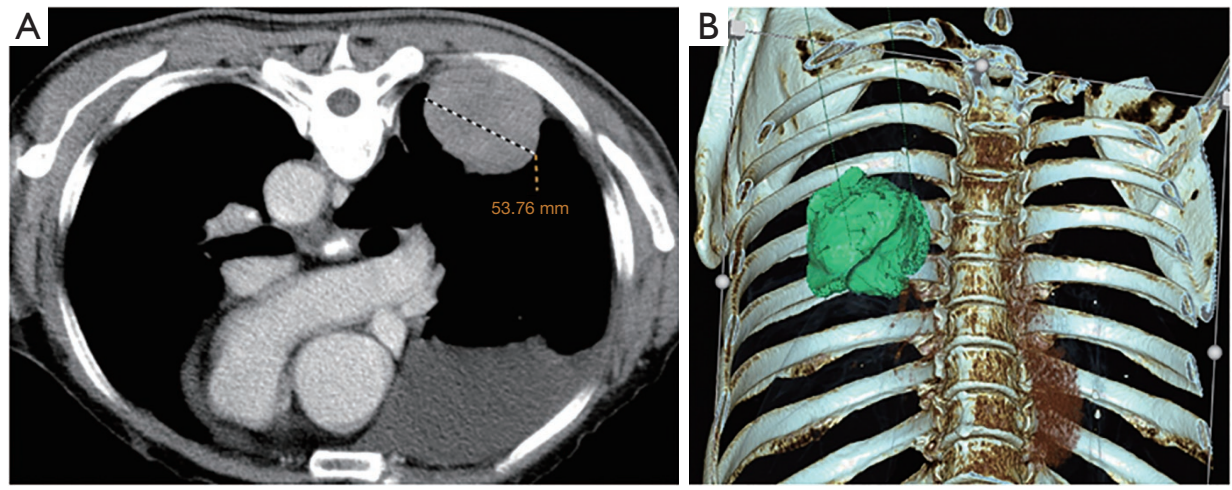

Figure 1 First enhanced chest CT. (A) The cross-section of contrast-enhanced chest CT in the prone position showed a solitary mass near the chest wall in the right lower pulmonary lobe. The maximum diameter of the mass was $53.76 \mathrm{~mm}$. The mass was slightly and uniformly enhanced after enhancement, and a medium amount of effusion was seen in the right thoracic cavity; (B) three-dimensional reconstruction intuitively showed the relative relationship between the tumor in the right lower pulmonary lobe, the ribs, and the spine. CT, computed tomography.

Although conventional surgical treatment is the major treatment for IMT (5-8), it is not suitable for elderly patients or those with severe cardiopulmonary insufficiency and nutritional disorders. For these patients, microwave ablation (MWA) can be used as a new treatment strategy. In the process of percutaneous MWA, a needle-like antenna is inserted percutaneously into the tumor under the image guidance of computed tomography (CT) or magnetic resonance imaging (MRI), and local microwave electromagnetic radiation is emitted from the probe's active tip, producing frictional tissue heating in a short time period that is capable of causing cell death by coagulation necrosis $(9,10)$. Usually, MWA systems use a 915 or $2,450 \mathrm{MHz}$ generator (11). To date, MWA monotherapy $(12,13)$ or the combination of MWA with chemotherapy (14), radiotherapy (15), or targeted therapy (16) has been widely applied in the treatment of lung cancer. However, the clinical application of MWA for the treatment of IMT has been rarely reported due to the rarity of IMT. Saenghirunvattana et al. (17) reported the case of a young patient (29 years old) with pulmonary IMT who achieved partial remission after receiving multiple treatments, including (but not limited to) three rounds of percutaneous MWA. Furthermore, no significant complications were observed during the perioperative period, which indicated that MWA monotherapy is probably applicable for the treatment of elderly patients with pulmonary IMT.

Herein, we report the case of an 80-year-old patient with pulmonary IMT. The maximum diameter of the tumor was $>5 \mathrm{~cm}$. The patient was successfully treated with three rounds of percutaneous MWA monotherapy and has no evidence of recurrence nearly 3 years later. We present the following article in accordance with the CARE reporting checklist (available at https://dx.doi.org/10.21037/tcr-211885).

\section{Case presentation}

An 80-year-old man presented to the hospital with a main complaint of fatigue for 2 months. Chest CT demonstrated a pulmonary mass in the right lower lobe and pleural effusion in the right thoracic cavity (Figure 1). The patient was in good physical condition previously; he had a history of hypertension and his blood pressure was well controlled by a hypotensor. He had been a heavy smoker for over 30 years and had quit smoking for nearly 20 years. His elder brother died of lung cancer; one sister had colon cancer; and one daughter had lung cancer. In addition to an anemic appearance, the physical examination results were non-specific. Laboratory findings revealed severe anemia (hemoglobin, $79 \mathrm{~g} / \mathrm{L}$ ), hypoproteinemia (albumin, $27.7 \mathrm{~g} / \mathrm{L}$ ), high erythrocyte sedimentation rate (ESR) $(97 \mathrm{~mm} / \mathrm{h})$, elevated hypersensitive C-reactive protein (CRP) $(99.4 \mathrm{mg} / \mathrm{L})$, and normal serum tumor marker levels. Red blood cell transfusion supplemented with albumin improved the patient's anemia and hypoalbuminemia. Meanwhile, B-ultrasound-guided thoracic tube placement and drainage of pleural effusion in the right thoracic cavity 

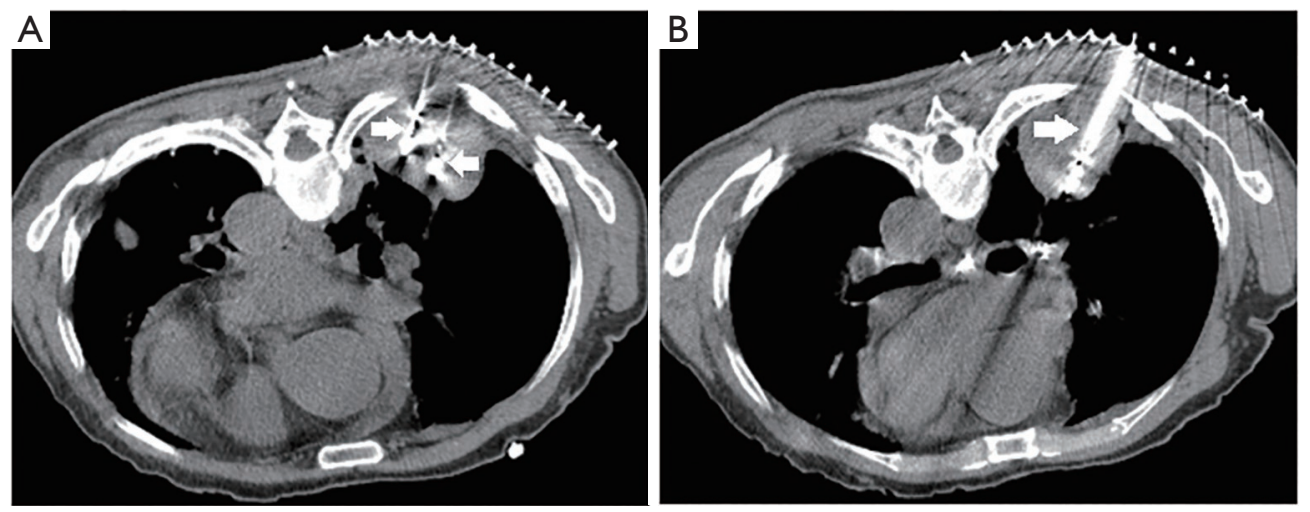

Figure 2 Needle CT images of the tumor in the right lower pulmonary lobe during the first MWA. Double-point ablation was adopted in the area below the tumor (A) and single-point ablation was adopted in the area above the tumor (B). The white arrow points to MWA needle. CT, computed tomography; MWA, microwave ablation.

was performed, and approximately $900 \mathrm{~mL}$ of hemorrhagic effusion was removed. The fluid subsequently became light yellow in color and clear, and the levels of carcinoembryonic antigen (CEA) in the pleural fluid $(3.67 \mathrm{ng} / \mathrm{mL})$ were normal. A pleural fluid smear showed that no cancer cells were present in the pleural fluid.

This patient could not underwent surgery due to his advanced age, anemia, and hypoproteinemia. The medical technology management committee of the Shaoxing Second Hospital approved this study of the MWA treatment. When his levels of hemoglobin $(95 \mathrm{~g} / \mathrm{L})$ and albumin $(29.9 \mathrm{~g} / \mathrm{L})$ were improved, CT-guided lung biopsy and MWA of the right lower lobe pulmonary mass were conducted on January 9th, 2018 (Figure 2).

Preoperative analgesia was performed by intramuscular injection of $100 \mathrm{mg}$ of pethidine 30 minutes before MWA. The patient was placed in the prone position and subjected to local anesthesia using a $1 \%$ lidocaine injection layer by layer up to the parietal pleura. A 16-G biopsy needle was used to obtain four pieces of $1.8 \mathrm{~cm}$ long dense lesion tissue for pathological examination. The ECO-100AL5 MWA needle was used, which was then connected to an ECO-100A1 Microwave Ablation System (ECO Medical Instrument Co., Ltd., Nanjing, Jiangsu, China). Multi-point ablation was performed; according to the manufacturer's instructions combined with texture of tumor, $55-\mathrm{W}$ radiation was applied and the total duration of ablation was 20 minutes. A water circulation cooling system was used to cool the surface temperature of the ablation needles. At the end of the procedure, the withdrawal path was ablated (Figure 2).
Subsequent histopathological examination revealed spindle cell proliferation and a mixed inflammatory infiltrate of lymphocytes and plasma cells (Figure $3 \mathrm{~A}$ ). The immunohistochemical staining showed that the cells were positive for vimentin, epithelial membrane antigen (EMA), actin (weakly+) and Ki-67 (1\%+), but negative for anaplastic lymphoma kinase (ALK) protein, smooth muscle actin (SMA), desmin, CD117, CD34, DOG-1, P53, pan-cytokeratin, high molecular weight cytokeratin (CKHMW), low molecular weight cytokeratin (CK-LMW) and S-100 protein (Figure 3B). The EML4-ALK fusion gene was also determined to be negative by real time polymerase chain reaction (PCR). Based on the biopsy pathology and clinical manifestations, the patient was diagnosed with pulmonary IMT. He was treated with glucocorticoids and diuretics to reduce tissue exudation, antibiotics to prevent infection, and expectorants and human blood albumin to correct hypoproteinemia. No complications occurred during the perioperative period and the patient was subsequently discharged (hemoglobin, $103 \mathrm{~g} / \mathrm{L}$; serum albumin, $35.0 \mathrm{~g} / \mathrm{L}$ ).

Regular follow-up was performed after MWA (Figure 4), and re-examination by enhanced chest CT indicated incomplete ablation of the tumor. CT-guided multi-point MWA of residual tumor in the right lower lobe were performed at 2 and 6 months (March 9th, 2018, and July 3 th, 2018, respectively) after the initial MWA procedure. The ablation power was $55 \mathrm{w}$ and the total duration of ablation was 24 and 18 minutes, respectively. The anesthesia method, ablation needle, ablation therapeutic instrument, and ablation position was identical to those 

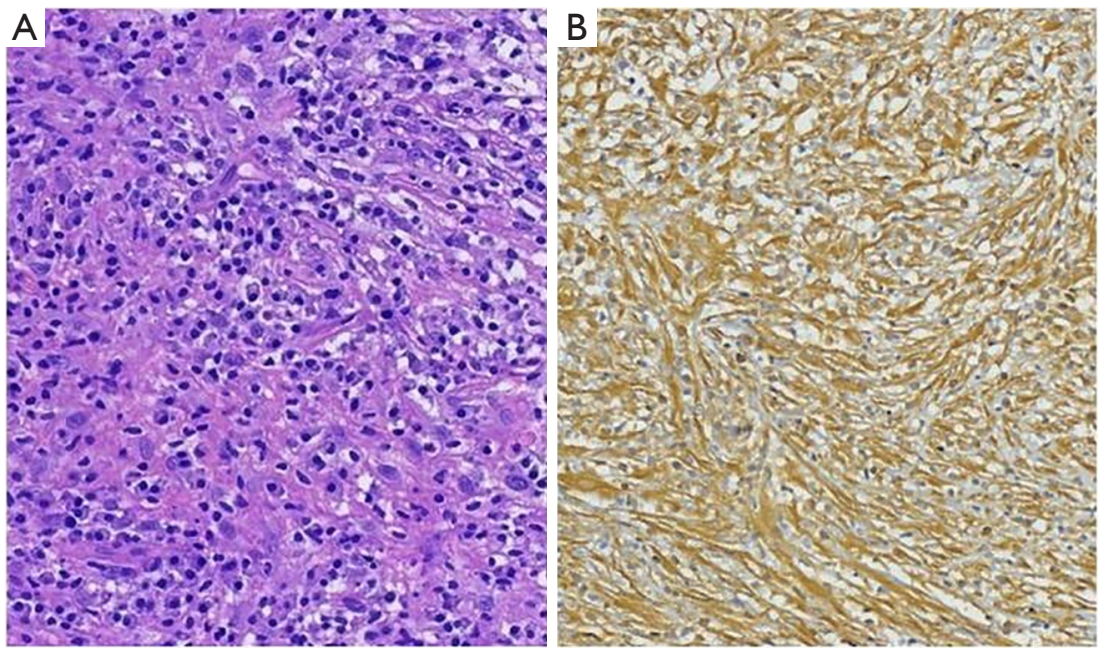

Figure 3 Pathological examination reconfirming pulmonary IMT. (A) Histological examination showed a spindle cell tumor with infiltration of lymphatic and plasma cells. Hematoxylin and eosin staining, original magnification $\times 100$. (B) Immunohistochemistry showed Vimentin positive, original magnification $\times 100$. IMT, inflammatory myofibroblastic tumor.
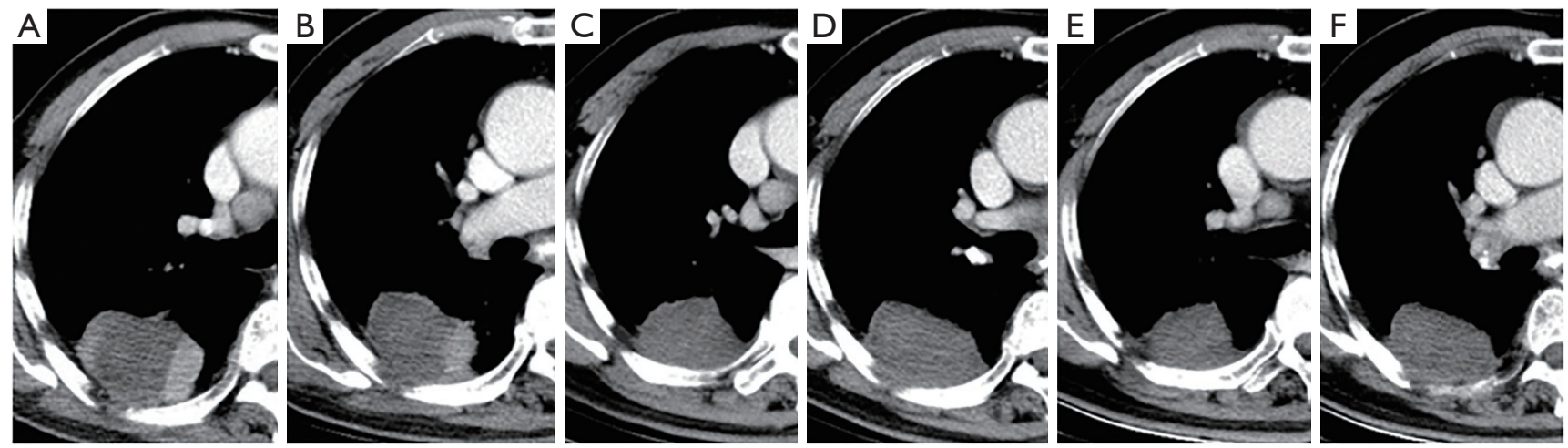

Figure 4 The enhanced chest CT was re-examined after WMA. CT images (A) and (B) were 2 months after the first ablation and 3 months after the second ablation, respectively. The contrast agents in the venous phase were locally enhanced without exception, suggesting incomplete local tumor ablation. (C) and (D) were conducted 3 months after the third ablation, while (E) and (F) were conducted 12 months after the third ablation. There was no enhancement of contrast agents during the venous phase, suggesting complete tumor ablation. CT, computed tomography; MWA, microwave ablation.

of the first ablation. No complications occurred during the perioperative period. The patient was followed up in the thoracic surgery outpatient department, and the latest enhanced chest CT (Figure 5) was conducted 35 months after the third ablation (June 18th, 2021). The contrast agent showed no signs of enhancement, suggesting that the tumor in the right lower pulmonary lobe was completely ablated. The patient was in good condition with stable weight, and had no fatigue, fever, cough, sputum, chest distress, shortness of breath, or other uncomfortable symptoms (hemoglobin, $134 \mathrm{~g} / \mathrm{L}$; serum albumin, $40.0 \mathrm{~g} / \mathrm{L}$; hypersensitive CRP, $5.8 \mathrm{mg} / \mathrm{L}$ ) (Figure 6).

All procedures performed in studies involving human participants were in accordance with the ethical standards of the institutional and/or national research committee(s) and with the Helsinki Declaration (as revised in 2013). Written informed consent was obtained from the patient for publication of this case report and accompanying images. A copy of the written consent is available for review by the editorial office of this journal. 

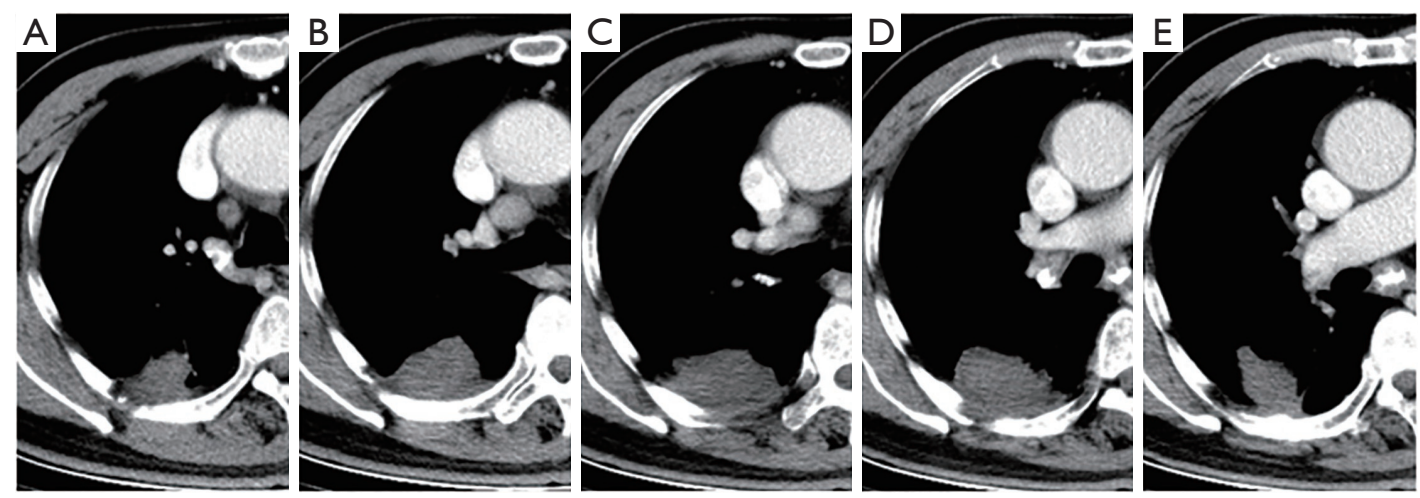

Figure 5 The latest re-examination of enhanced chest CT 35 months after the third ablation, suggesting complete ablation of the tumor in the right lower lobe. CT, computed tomography.

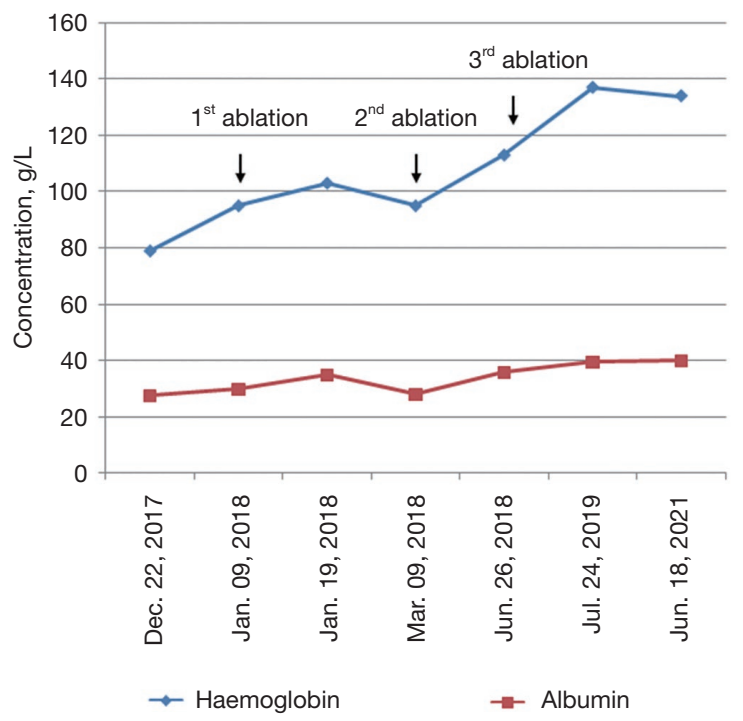

Figure 6 Timeline and dynamic changes of major laboratory examinations.

\section{Discussion}

IMT was previously considered a benign and inflammatory disease with good prognosis in most cases. However, IMT is currently considered a rare neoplastic disease due to its characteristics of aneuploidy cells (18), possession of tumor stem cells (19), and multiple types of gene mutations. Therefore, although IMT generally presents benign biological behavior, some IMT patients may develop local recurrence or even distant metastasis. The recurrence rate of IMT at different anatomical sites varies, ranging from less than $2 \%$ for pulmonary lesions to $25 \%$ for extrapulmonary lesions, and the rate of distant metastasis is less than $5 \%(2)$. The lungs and brain are the most common sites for metastasis, followed by bone and liver (2). It has been reported that the tongue (20), kidney (21), and uterus (22) are also target organs for metastasis in IMT. $A L K$ gene $2 \mathrm{p} 23$ rearrangement occurs in approximately $50 \%$ of metastasis cases (23), resulting in overexpression of the ALK protein, but the pathogenesis of ALK expression-lacking IMT remains unknown. A study based on next-generation sequencing (NGS) assays reported that two of nine ALKnegative patients harbored PDGFR $\beta$ kinase fusions, and four of them harbored ROS1 kinase fusions, suggesting that IMT is likely a kinase fusion-driven tumor (24).

Clinically, it is very difficult to distinguish pulmonary IMT from lung cancer (25). The clinical manifestations of IMT are not specific. Most patients with pulmonary IMT have no obvious symptoms. Some patients may develop cough, sputum, chest tightness, chest pain and other nonspecific respiratory symptoms. Some patients may also present with fever, anemia, fatigue, poor appetite, dizziness, weight loss, and other systemic symptoms. Laboratory examination demonstrates elevated hypersensitive CRP, elevated ESR, anemia, and hypoproteinemia. In most cases, chest CT images exhibit clear edges and uniform density of masses in the periphery of lung. Enhanced chest CT images demonstrate mild, moderate, or even significant enhancement, as well as a marked increase in fluorodeoxyglucose (FDG) metabolism (25). It has been reported that $15-30 \%$ of patients probably present with "inflammatory syndrome", which is characterized by fever, fatigue, and weight loss, while laboratory evaluation shows anemia, elevated ESR and CRP, thrombocytosis, and/or polyclonal hypergammaglobulinemia, which is considered to be related to secretion of interleukin (IL)-6 (23). This 
syndrome disappears after tumor resection, but reoccurs with tumor recurrence (1).

Our patient reported fatigue at admission, and the laboratory examination suggested anemia $(79 \mathrm{~g} / \mathrm{L})$ and increased hypersensitive CRP $(99.4 \mathrm{mg} / \mathrm{L})$. After treatment, the fatigue symptom disappeared, and hemoglobin and hypersensitive CRP return to normal (hemoglobin, $134 \mathrm{~g} / \mathrm{L}$; hypersensitive CRP, $5.8 \mathrm{mg} / \mathrm{L}$ ). On admission, our patient had right pleural effusion, and approximately $900 \mathrm{~mL}$ of bloody pleural effusion was removed after thoracic catheter drainage. The bloody pleural effusion related to the local invasion of tumor cells in the chest wall was consistent with the biological behavior of a malignant neoplasm.

In 2013, the World Health Organization (WHO) defined IMT as a tumor composed of myofibroblastic and fibroblastic spindle cells, accompanied by a large amount of inflammatory cells (such as plasma cells, lymphocytes, and/or eosinophils) (1). For this patient, a 16-G biopsy needle was used to obtain four pieces of $1.8 \mathrm{~cm}$ long dense lesion tissue for pathological examination. Histological examination showed a spindle cell tumor with infiltration of lymphatic and plasma cells. Immunohistochemistry indicated vimentin positive and actin weakly positive. Combined with patient's clinical features and local aggressiveness, he was diagnosed with pulmonary IMT.

Previously, conventional surgery (thoracotomy or videoassisted thoracoscopic surgery) was the main therapeutic strategy for pulmonary IMT $(5,6,26,27)$. Complete resection probably results in cure $(7,8)$. For patients with unresectable IMT, a variety of palliative methods can be adopted for tumor control, including glucocorticoids $(28,29)$, nonsteroidal anti-inflammatory drugs $(30,31)$, macrolide antibiotics (32), chemotherapy/radiotherapy (33-35), anti-angiogenic drugs (36), targeted therapy (24), etc. For approximately $50 \%$ patients with anaplastic lymphoma kinase (ALK) positive IMT, ALK inhibitor (crizotinib) treatment is suitable $(23,34,37,38)$. Furthermore, treatment of pulmonary IMT accompanied by ROS1 rearrangement with crizotinib can achieve sustained remission (5). This suggests the need for more extensive molecular diagnostics, including NGS, to identify more potential therapeutic targets for the treatment of ALK-negative pulmonary IMT.

Since the Chinese scholar Feng et al. (39) took the lead in the application of MWA for the treatment of lung cancer in 2002, percutaneous MWA has been widely applied to treat medically inoperable patients with lung cancer, and the relevant expert consensus and standards has been published (40,41). Wang et al. (42) compared the efficacy and treatment expense between percutaneous MWA and thoracoscopic lobectomy in patients with stage I non-small cell lung cancer (NSCLC). They reported that the 1-year overall survival (OS) rates of the MWA and lobectomy groups were $97.82 \%$ and $96.65 \%$, respectively, and the 2-year OS rates were $91.30 \%$ and $90.59 \%$, respectively. Thus, there were no significant differences between these two methods, while MWA could shorten the duration of hospitalization and reduce the medical costs. Pusceddu et al. (43) evaluated the clinical outcome of CT-guided MWA in patients with large-lesion NSCLC (tumor size: $5.0 \pm 1.8 \mathrm{~cm}$ ). They found that $18.5 \%$ of patients achieved complete tumor necrosis after two rounds of MWA, and $4.6 \%$ of patients achieved complete tumor necrosis after three rounds of MWA. The 1-, 2-, 3-, and 5-year OS rates were $78.2 \%, 48.3 \%, 34.8 \%$, and $18.3 \%$, respectively.

In this case, our patient was not able to undergo conventional lobectomy surgery due to his advanced age, anemia, and hypoalbuminemia. After extensive treatment, CT-guided percutaneous multipoint MWA was conducted under local anesthesia three rounds with an ablation power of $55 \mathrm{w}$. The total ablation time of the first, second, and third rounds were 20,24, and 18 minutes, respectively. The ablation power used for our patient was equivalent to that used for conventional lung cancer patients, and the ablation time was even longer than that for conventional lung cancer patients, which might be attributed to the highdensity of the lesion tissue rich in myofibroblastic spindle cells, suggesting that higher ablation power and longer ablation time may be needed for complete ablation of dense tumors. For tumors with the maximum diameter of over $5 \mathrm{~cm}$, more rounds of treatment and multiple ablation points are required for curative ablation. The treatment period for our patient was 6 months long and no distant metastasis occurred during the treatment, which was consistent with previous reports showing that IMT has of low degree of malignance and most patients have a good prognosis (27). For our patient, although the diameter of the tumor was over $5 \mathrm{~cm}$, he was successfully treated with MWA monotherapy and has no evidence of recurrence nearly 3 years since the last treatment according to the follow-up chest enhancement CT images, laboratory examination, and clinical manifestations. This result suggests that low-grade malignant tumors, such as pulmonary IMT, are likely cured by multiple ablations (even large volume tumors), which expands the indications of curative ablation (40).

Because of its advantages of ultra-minimal invasion, repeatability, no bone marrow suppression, lower cost, 
shorter ablation times, larger ablation zones and lower heat sink effect, microwave ablation achieves good efficacies of the tumor patients with poor cardiopulmonary function, advanced age, refusing surgical resection or recurrent single lesions. However, when the tumor is too large, closing to the large airway or blood vessel, the application of microwave ablation is limited because of its high risk and easy residue.

\section{Conclusions}

In conclusion, pulmonary IMT is a rare neoplastic lesion, which has a potential for recurrence and metastasis. Percutaneous MWA monotherapy may be an important alternative strategy for medically inoperable patients.

\section{Acknowledgments}

The authors would like to thank Dr. Yi Yu, Department of Life Sciences Institute, Zhejiang University (Zhejiang, China) for English language editing.

Funding: None.

\section{Footnote}

Reporting Checklist: The authors have completed the CARE reporting checklist. Available at https://dx.doi. org/10.21037/tcr-21-1885

Conflicts of Interest: All authors have completed the ICMJE uniform disclosure form (available at https://dx.doi. org/10.21037/tcr-21-1885). The authors have no conflicts of interest to declare.

Ethical Statement: The authors are accountable for all aspects of the work in ensuring that questions related to the accuracy or integrity of any part of the work are appropriately investigated and resolved. All procedures performed in studies involving human participants were in accordance with the ethical standards of the institutional and/or national research committee(s) and with the Helsinki Declaration (as revised in 2013). Written informed consent was obtained from the patient for publication of this case report and accompanying images. A copy of the written consent is available for review by the editorial office of this journal.

Open Access Statement: This is an Open Access article distributed in accordance with the Creative Commons Attribution-NonCommercial-NoDerivs 4.0 International License (CC BY-NC-ND 4.0), which permits the noncommercial replication and distribution of the article with the strict proviso that no changes or edits are made and the original work is properly cited (including links to both the formal publication through the relevant DOI and the license). See: https://creativecommons.org/licenses/by-nc-nd/4.0/.

\section{References}

1. Fletcher CD, Bridge JA, Hogendoorn PC, et al. World Health Organization classification of soft tissue and bone tumours. Lyon: IARC Press, 2013.

2. Gleason BC, Hornick JL. Inflammatory myofibroblastictumours: where are we now? J Clin Pathol2008;61:428-37.

3. Patnana M, Sevrukov AB, Elsayes KM, et al. Inflammatory pseudotumor: the great mimicker. AJR Am J Roentgenol 2012;198:W217-27.

4. Sagar AES, Jimenez CA, Shannon VR. Clinical and Histopathologic Correlates and Management Strategies for Inflammatory Myofibroblastic tumor of the lung. A case series and review of the literature. Med Oncol 2018;35:102.

5. Mai S, Xiong G, Diao D, et al. Case report: Crizotinib is effective in a patient with ROS1-rearranged pulmonary inflammatory myofibroblastic tumor. Lung Cancer 2019;128:101-4.

6. Sziklavari Z, Droste A, Neu R, et al. Surgical Treatment of Pseudotumours of the Lung. Zentralbl Chir2018;143:90-5.

7. Fabre D, Fadel E, Singhal S, et al. Complete resection of pulmonary inflammatory pseudotumors has excellent long-term prognosis. J Thorac Cardiovasc Surg 2009; 137:435-40.

8. Wang FH, Liang JH, Zeng JH, et al. Clinical characteristics, diagnosis and treatment of pulmonary inflammatory myofibroblastic tumor in children. Zhonghua Zhong Liu Za Zhi 2017;39:299-302.

9. Abbas G. Microwave ablation. Semin Thorac Cardiovasc Surg 2011;23:81-3.

10. Ahmed M, Solbiati L, Brace CL, et al. Image-guided tumor ablation: standardization of terminology and reporting criteria--a 10-year update. Radiology 2014;273:241-60.

11. Ward RC, Healey TT, Dupuy DE. Microwave ablation devices for interventional oncology. Expert Rev Med Devices 2013;10:225-38.

12. Nance M, Khazi Z, Kaifi J, et al. Computerized 
tomography-Guided Microwave Ablation of Patients with Stage I Non-small Cell Lung Cancers: A Single-Institution Retrospective Study. J Clin Imaging Sci 2021;11:7.

13. Tsakok MT, Little MW, Hynes G, et al. Local control, safety, and survival following image-guided percutaneous microwave thermal ablation in primary lung malignancy. Clin Radiol 2019;74:80.e19-26.

14. Zhang YQ, Wu YL, Feng Y, et al. A Clinical Study on Microwave Ablation in Combination with Chemotherapy in Treating Peripheral IIIB-IV Nonsmall Cell Lung Cancer. Cancer Biother Radiopharm 2020. [Epub ahead of print]. doi: 10.1089/cbr.2020.3859.

15. Xu X, Ye X, Liu G, et al. Targeted percutaneous microwave ablation at the pulmonary lesion combined with mediastinal radiotherapy with or without concurrent chemotherapy in locally advanced non-small cell lung cancer evaluation in a randomized comparison study. Med Oncol 2015;32:227.

16. Ni Y, Ye X, Yang X, et al. Microwave ablation as local consolidative therapy for patients with extracranial oligometastatic EGFR-mutant non-small cell lung cancer without progression after first-line EGFR-TKIs treatment. J Cancer Res Clin Oncol 2020;146:197-203.

17. Saenghirunvattana S, Napairee C, Geraplangsub S. Microwave Ablation in Managing Lung Cancer: A Case Report. Bangk Med J 2019;15:73.

18. Biselli R, Ferlini C, Fattorossi A, et al. Inflammatory myofibroblastic tumor (inflammatory pseudotumor): DNA flow cytometric analysis of nine pediatric cases. Cancer 1996;77:778-84.

19. Masciale V, Grisendi G, Banchelli F, et al. Cancer StemLike Cells in a Case of an Inflammatory Myofibroblastic Tumor of the Lung. Front Oncol 2020;10:673.

20. Rodrigues C, Cabral D, Almodovar T, et al. Unusual Behavior of a Lung Inflammatory Myofibroblastic Tumor: Case Report. Rev Port Cir Cardiotorac Vasc 2017;24:140.

21. Na YS, Park SG. Inflammatory myofibroblastic tumor of the pleura with adjacent chest wall invasion and metastasis to the kidney: a case report. J Med Case Rep 2018;12:253.

22. Zhang J, Li Y, Lou L. Uterine metastases originating from a pulmonary inflammatory myofibroblastic tumor. J Cancer Res Ther 2018;14:S257-9.

23. Surabhi VR, Chua S, Patel RP, et al. Inflammatory Myofibroblastic Tumors: Current Update. Radiol Clin North Am 2016;54:553-63.

24. Lovly CM, Gupta A, Lipson D, et al. Inflammatory myofibroblastic tumors harbor multiple potentially actionable kinase fusions. Cancer Discov 2014;4:889-95.
25. Sonomura T, Hasegawa S, Takeuchi H, et al. Inflammatory myofibroblastic tumor of the lung indistinguishable from adenocarcinoma on imaging studies. Clin Nucl Med 2014;39:740-1.

26. Sakurai H, Hasegawa T, Watanabe Si, et al. Inflammatory myofibroblastic tumor of the lung. Eur J Cardiothorac Surg 2004;25:155-9.

27. Zhang N, Zeng Q, Chen C, et al. Clinical characteristics and prognosis of pulmonary inflammatory myofibroblastic tumor: An over 10-year retrospective analysis. Pediatr Investig 2020;4:192-7.

28. Shimodaira Y, Sugawara K, Fukuda S, et al. Aggressive Inflammatory Myofibroblastic Tumor without Anaplastic Lymphoma Kinase Gene Rearrangement in the Rectum with Liver Metastasis. Intern Med 2020;59:495-9.

29. Ikeda T, Nakano J, Kushida Y, et al. Multiple Pulmonary Inflammatory Myofibroblastic Tumor. Kyobu Geka 2019;72:367-70.

30. Moon CH, Yoon JH, Kang GW, et al. A case of recurrent pulmonary inflammatory myofibroblastic tumor with aggressive metastasis after complete resection. Tuberc Respir Dis (Seoul) 2013;75:165-9.

31. Chavez C, Hoffman MA. Complete remission of ALK-negative plasma cell granuloma (inflammatory myofibroblastic tumor) of the lung induced by celecoxib: A case report and review of the literature. Oncol Lett 2013;5:1672-6.

32. Watanabe H, Uruma T, Tazaki G, et al. Remission of ALK-negative primary pulmonary inflammatory myofibroblastic tumor on treatment with clarithromycin: A case report and review of the literature. Oncol Lett 2016;11:1757-61.

33. Gaudichon J, Jeanne-Pasquier C, Deparis M, et al. Complete and Repeated Response of a Metastatic ALKrearranged Inflammatory Myofibroblastic Tumor to Crizotinib in a Teenage Girl. J Pediatr Hematol Oncol 2016;38:308-11.

34. Kimbara S, Takeda K, Fukushima H, et al. A case report of epithelioid inflammatory myofibroblastic sarcoma with RANBP2-ALK fusion gene treated with the ALK inhibitor, crizotinib. Jpn J Clin Oncol 2014;44:868-71.

35. Kube S, Vokuhl C, Dantonello T, et al. Inflammatory myofibroblastic tumors-A retrospective analysis of the Cooperative WeichteilsarkomStudiengruppe. Pediatr Blood Cancer 2018;65:e27012.

36. Liu Q, Wei J, Liu X, et al. Anaplastic lymphoma kinasenegative pulmonary inflammatory myofibroblastic tumor with multiple metastases and its treatment by Apatinib: A 
case report. Medicine (Baltimore) 2019;98:e18414.

37. Theilen TM, Soerensen J, Bochennek K, et al. Crizotinib in ALK+ inflammatory myofibroblastic tumors-Current experience and future perspectives. Pediatr Blood Cancer 2018. doi: 10.1002/pbc.26920.

38. Gambacorti-Passerini C, Orlov S, Zhang L, et al. Longterm effects of crizotinib in ALK-positive tumors (excluding NSCLC): A phase 1b open-label study. Am J Hematol2018;93:607-14.

39. Feng $W$, Liu $W$, Li C, et al. Percutaneous microwave coagulation therapy for lung cancer. Zhonghua Zhong Liu Za Zhi 2002;24:388-90.

40. Ye X, Fan W, Wang H, et al. Expert consensus workshop report: Guidelines for thermal ablation of primary and metastatic lung tumors (2018 edition). J Cancer Res Ther 2018;14:730-44.

40. Ye X, Fan W, Wang H, et al. Expert consensus workshop report: Guidelines for thermal ablation of primary and metastatic lung tumors (2018 edition). J Cancer Res Ther2018;14:730-44.

41. Venturini M, Cariati M, Marra P, et al. CIRSE Standards of Practice on Thermal Ablation of Primary and Secondary Lung Tumours. Cardiovasc Intervent Radiol 2020;43:667-83.

42. Wang Y, Liu B, Cao P, et al. Comparison between computed tomography-guided percutaneous microwave ablation and thoracoscopic lobectomy for stage I nonsmall cell lung cancer. Thorac Cancer 2018;9:1376-82.

43. Pusceddu C, Melis L, Sotgia B, et al. Usefulness of percutaneous microwave ablation for large non-small cell lung cancer: A preliminary report. Oncol Lett 2019;18:659-66.

(English Language Editor: A. Kassem)

Cite this article as: Zhang Y, Zheng G, Meng X, Li Y, Shi D, Yu J. Microwave ablation for the management of pulmonary inflammatory myofibroblastic tumor: a case report and literature review. Transl Cancer Res 2021;10(10):4582-4590. doi: $10.21037 /$ tcr-21-1885 